\title{
Peripheral osteoma of the mandible -an unusual presentation
}

\author{
Sai Sravanthi Gadwalwari1,", Santan Reddy Muthyam², Ramlal Gantala ${ }^{3}$, Rama Raju D ${ }^{4}$, \\ Shrinidhi Reddy Banda ${ }^{5}$ \\ ${ }^{1,5}$ Senior Lecturer, ${ }^{2}$ Associate Professor and HOD, ${ }^{1,2,5}$ Dept. of Oral Medicine and Radiology, Sri Balaji Dental College, \\ Hyderabad, Telangana, ${ }^{3}$ Professor and HOD, ${ }^{4}$ Professor, Dept. of Oral Medicine and Radiology, SVS Institute of Dental \\ Sciences, Mahbubnagar, Telangana, India
}

*Corresponding Author: Sai Sravanthi Gadwalwari

Email: saijhumky@gmail.com

\begin{abstract}
Osteoma is a benign osteogenic, mostly asymptomatic tumour characterized by proliferation of cancellous/compact bone. It is one of the rare entity with $0.01-0.4 \%$ prevalence, and $2.9 \%$ of all tumors; $12.1 \%$ of all benign tumors. This could be Central, Peripheral, or Extra-skeletal in location mostly occurring in the Cranio-facial bones. Peripheral osteomas are rare to occur in the jaws with predilection to mandibular lingual aspect of body, angle, and inferior border. We here report a case of 15 year boy with swelling in front of left ear. Clinical and radiographic examination brought us to a working diagnosis as Osteoma in the buccal aspect of left ramus of mandible, which is huge in size. Thorough examination is done to rule out any syndromes associated with it, such as Gardner's Syndrome. Following which surgical excision is done under general anesthesia, and an 8 months regular follow-up reveals no recurrence.
\end{abstract}

Keywords: Osteoma, Osteogenic, Benign.

\section{Introduction}

Osteoma is a rare, benign, osteogenic tumour with prevalence mostly in the craniofacial region. In mandible, it is most predominant in body, condyle, and angle. We here report a case of large peripheral osteoma on facial aspect of left ramus, which is the rarest site of occurrence and this case takes its uniqueness by its presentation on the buccal aspect of ramus of the mandible.

A 15 year old male patient presented with a swelling in front of left ear since 3 months, which was initially small and then gradually progressed to present size. (Fig. 1A). This swelling is not associated with pain and no history of any physical trauma in that region. His past medical and dental histories were not contributory. Clinical examination revealed a solitary extra oral swelling on the left mandibular ramus region in front of the ear; which is oval in shape, $3 \times 3.5 \mathrm{~cm}$ in diameter, non-tender, immobile and bony hard in consistency (Fig. 1B). Intra-oral examination revealed retained 55, 85, and buccally erupting 15 . Intraoral swelling is not clearly appreciated (Fig. 2), but a stony hard mass is palpable in the left ramus region.

Differential Diagnosis: Correlating the clinical and radiographic features, a provisional diagnosis of osteoma of the left buccal side of ramus was made. Correlating the clinical and radiographic findings, bony exostosis, complex odontoma, and osteoid osteoma were considered as differential diagnosis.

Investigations: Pantomograph reveals retained 55 and 85 , with erupting 15 and 45 . A well defined radioopaque mass of size $3 \times 4 \mathrm{~cm}$ is noticed in the left ramus region, completely occupying the ramus medio-laterally and extending $0.2 \mathrm{~cm}$ above the sigmoid notch till distal surface of 38 (Fig. 3).
Computed tomography axial and 3D reconstruction view reveals a well defined hyperdense pedunculated mass arising from the left ramus, and completely occupying it (Fig. 4,5). No noticeable abnormalities noticed in haematological investigations. Whole abdomen ultrasound revealed no intestinal polyps. Complete Excisional biopsy was done under general anesthesia, which exhibits dense bony trabaculae showing lacunae with osteocytes and little stroma. Haversian canals with their associated lamella and lacunae, along with Osteoblastic rimming is noted which is suggestive of osteoma (Fig. 6 A,B).

Treatment: After a thorough pre-anaesthetic evaluation, and taking consent from both patient and parents, complete surgical excision was done under general anesthesia through intra-oral approach. After which, the patient is kept under antibiotic coverage along with physiotherapy. Complete healing was noticed in 20 days.

Outcome and followup: Subject is under constant periodic check up for 8 months, with radiographic evaluation revealed normal bone formation with no recurrence (Fig. 7). Subject is happy with a confident and normal facial appearance.

\section{Discussion}

Osteomas are rare benign tumours of osteogenic origin, characterized by proliferation of bony tissue. ${ }^{1}$ Osteomas are characterised by proliferation of cancellous/compact bone. These are categorised into three types, Central, Peripheral, and Extra-skeletal type. Central osteoma arise from endosteum, peripheral osteoma from periosteum, and extra-skeletal osteomas occur in soft tissues. ${ }^{2}$ Etiopathogenesis of osteoma remains obscure, different school of thought have put 
forward various etiopathological reports which are reactive mechanism to some unrecognised trauma by sub-periosteal bleeding and muscle traction, developmental anomaly, or might be some neoplasm..$^{3-5}$

Osteomas are more prevalent in the craniofacial region accounting mostly in the paranasal sinuses, followed by ethemoid sinuses, orbital wall, temporal bone, pterygoid processes, and external ear canal. Occurrence of osteoma in mandible is considered rare, with predominant in posterior body, condyle, angle, ascending ramus lingual, coronoid process, anterior body, and sigmoid notch in decreasing order, with our case reported in the buccal aspect of ramus by taking its unique entity. Age of occurrence of osteoma is ranging from 11 years to 73 years, mostly occurring in $2^{\text {nd }}$ and $3^{\text {rd }}$ decades. Generally these are considered without any gender predilection but there are some studies which have shown results with more female predilection $1.5: 1,{ }^{6}$ with our case reported in male.

Osteomas are benign, Painless, slow growing, unilateral, pedunculated/sessile, mushroom like, nonfluctuant, non-compressable masses. In many cases these peripheral osteomas are incidental finding, however sometimes may be the main problem based on the location of the osteoma by causing deformities of face or by difficulty in functioning of the oral cavity, headache or exopthalmos. Size of peripheral osteoma varies from $10 \mathrm{~mm}$ to $40 \mathrm{~mm} .^{2,7}$

Subjects with multiple osteomas must be evaluated for syndromes associated with such as Gardner's syndrome. This syndrome is a triade having multiple impacted teeth or supernumerary teeth, multiple osteomas (hard/soft tissue), and gastrointestinal polyps. Osteomas through a light on the investigation of colorectal polyps as these osteomas are early to occur in the gastrointestinal polyps, thus a dentist plays an important role in diagnosing the polyps.
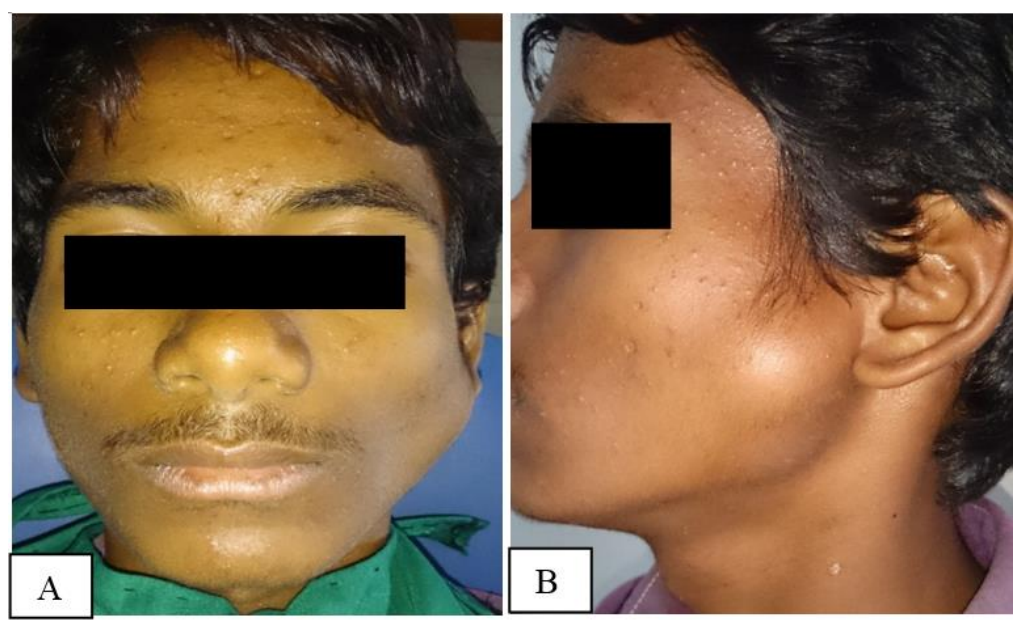

Fig. 1A: Showing the asymmetry on the left side of face due to swelling in-front of the ear; 1B: showing the size of the swelling
Radiographically osteoma appeasr as a uniformly radiopaque structure, with well defined borders, and at times may displace the adjacent muscle tissue and cause dysfunction. ${ }^{8}$ Bonder L et al., (1998) and Kashima et al., (2000) proposed that computed tomography is the best method for evaluation of osteoma than conventional radiography.

Osteomas are best treated by surgical excision. Surgical approach whether intra-oral or extra-oral is the choice and decision made by the operator based on the location and size of the osteoma. Fernando Kendi Horikawa (2012) said that intra-oral approach is preferred for preservation of facial nerve. But Longo et al., (2010) advised extra-oral approach if the osteoma is large and located posteriorly.

Recurrence of osteoma is very rare..$^{9}$ One case in the literature has shown recurrence after 9 years treated by Bosshardt et al., (1971). So periodic checkups annually are to be performed. ${ }^{10}$

Take home points:

1. Occurrence of osteoma in the buccal aspect of mandibular ramus is rare, here in we report a case of osteoma with its unique entity of occurrence on mandibular ramus region.

2. Multiple osteomas should be evaluated for Gardner's syndrome, which is the early feature of gastrointestinal polyps. Where some adenomas might progress to malignancy.

3. Based on the location of the osteoma the complaints of the subject may vary, from asymptomatic to sever pain and dysfunction.

4. Radiographically computed tomography is the best modality to evaluate osteoma in location, size, and effect on surrounding tissues, than conventional radiographs. 


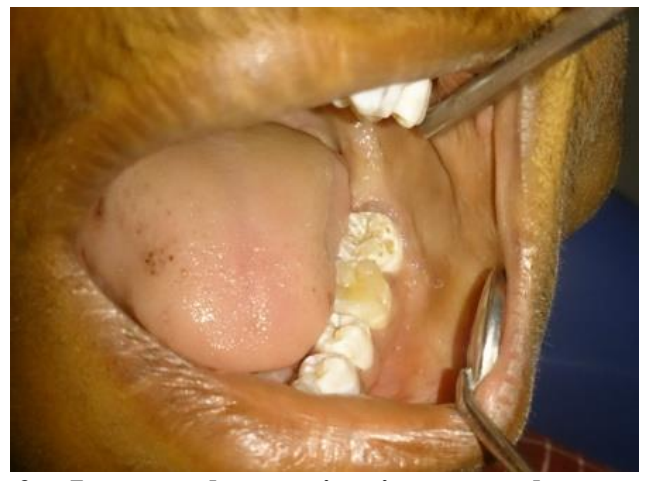

Fig. 2: Intra-oral examination reveals no clear evident swelling

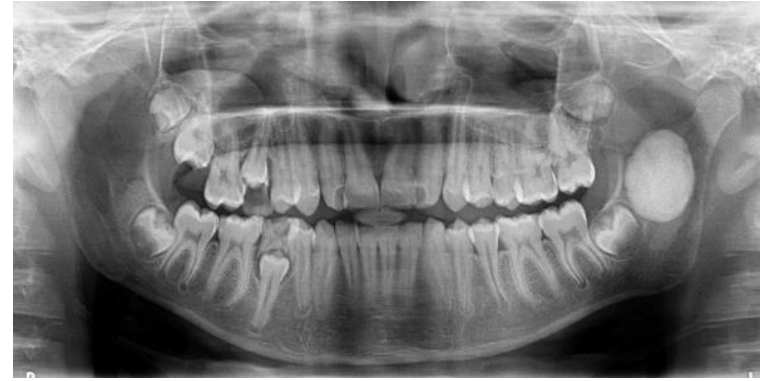

Fig. 3: Orthpoantomograph reveals permanent set of dentition with retained 55,85 and erupting 15 , and a well defined radiolucency in the left ramus extending from the sigmoid notch superiorly till $3.5 \mathrm{~cm}$ inferiorly from it

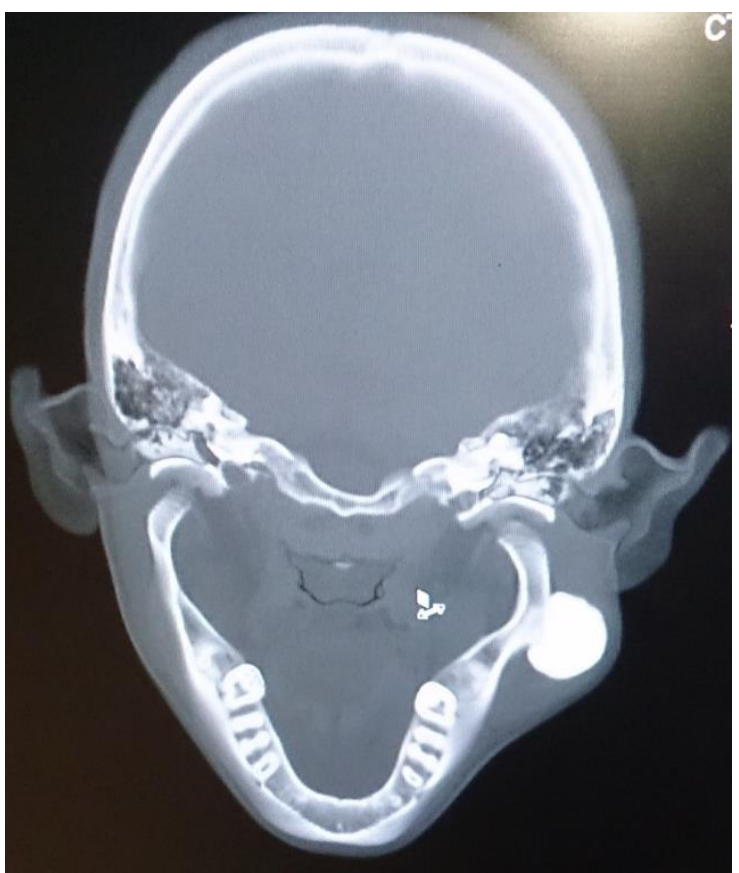

Fig. 4: axial Computed Tomography reveals a well defined hyperdense area arising from the ramus of the mandible

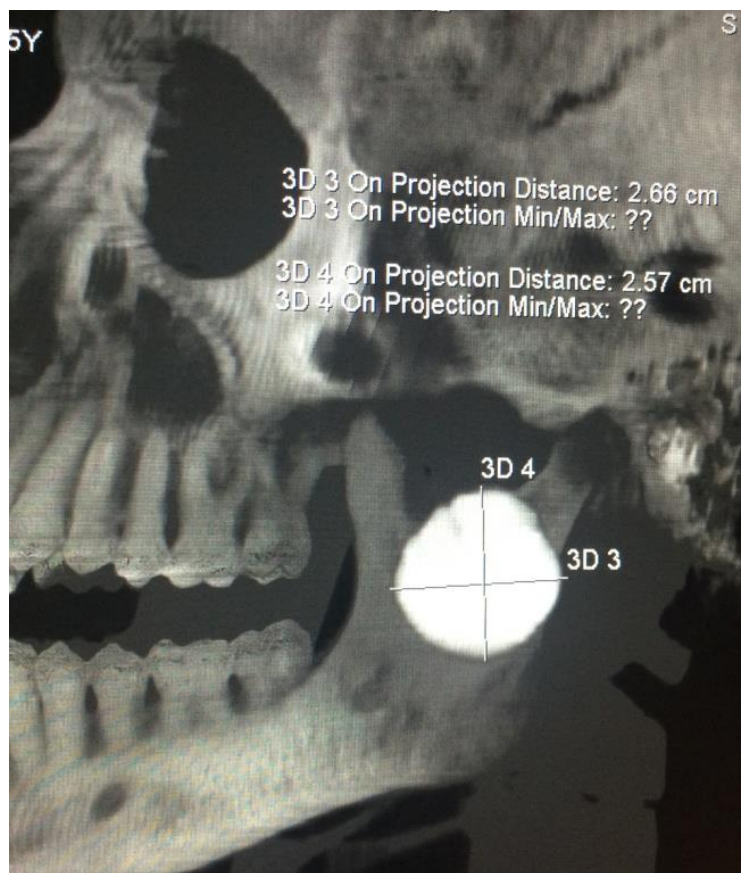

Fig. 5: 3D reconstruction view reveals extent of the swelling occupying the complete ramus of the mandible on the left side

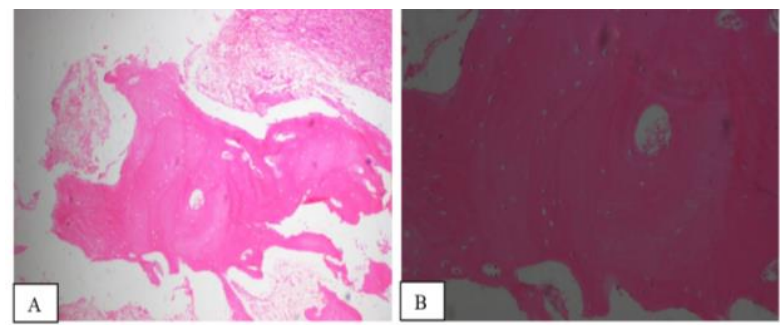

Fig. 6A: photomicrograph showing dens cortical bone with haversian canals and lacunae with osteocytes; 6B: photomicrograph showing lamellated bone and lacunae with osteocytes

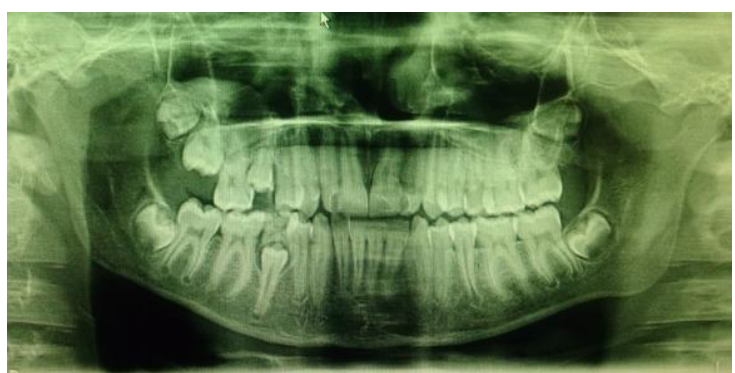

Fig. 7: Orthopantomography 8 months after surgery showing complete healing of bone and no recurrence

\section{References}

1. Fernando KH, Ronaldo RDF, Fernando AM, Antonio JG. Peripheral osteoma of the maxillofacial region a study of 10 cases. Braz J Otorhinolaryngol. (2012);78-5.

2. Emel B, Aydan A, Bora O, and Omer G. "Large Peripheral Osteoma of the Mandible: A Case Report. Int $J$ Dent. (2010), Article ID 834761. 
3. Soheyl S, Shambulingappa P, Amit A, Ravinder S, Neha B, Gaurav G. Peripheral osteomas of the mandible - a case report. JOHR. 2011;2(4).

4. Yitzhak W, Michael N, Lipa B "Peripheral osteoma of the maxillofacial region. Diagnosis and management: A study of 14 cases. Med Oral Patol Oral Cir Bucal. 2005;10-12 ;E139-42.

5. Dr. Gururaju C.R, Dr. Usha C "Peripheral Osteoma of Mandible. IOSR Journal of Dental and Medical Sciences (IOSR-JDMS), 2014;13-5;73-76.

6. Yitzhak W, Michael N, Lipa Bodner, "Peripheral osteoma of the maxillofacial region. Diagnosis and management: A study of 14 cases. Med Oral Patol Oral Cir Bucal. 2005;10-12:139-42.

7. Talita RTDF, Luiz AMG, Jurema FLDC, Ivson CJCL, Danyel EDCP. Solitary peripheral osteomas of the jaws. Imaging Sci Dent. 2012;42:99-103.
8. Stuart C. White, Michael J. Pharoah. Oral Radiology Principles and Interpretation; First South Asia Edition.

9. Kashima K, Rahman OI, Sakoda S, Shiba R. "Unusual peripheral osteoma of the mandible: report of 2 cases." $J$ Oral Maxillofac Surg. 2000:58-8:911-3.

10. Bosshardt L, Gordon RC, Westerberg M, Morgan A. "Recurrent peripheral osteoma of mandible: report of case. J Oral Surg. 1971;29-6:446-50. 